Proceedings

\title{
A descriptive study of the differences in the type of admissions of the hospital stay in a Psychiatric Hospitalization Unit be- tween 2019 and $2020^{\dagger}$
}

\author{
Marta Llorente-Alonso ${ }^{1,2^{*}}$, Gumersindo Tirado ${ }^{3}$, Cristina García-Ael $^{4}$ and Gabriela Topa ${ }^{4}$ \\ 1 Health Psychology Program, International School of Doctorate, National Distance Education University \\ (UNED), C/Bravo Murillo,38, 3ª , 28015, Madrid; mllorente78@alumno.uned.es \\ 2 Servicio de Psiquiatría y Salud Mental, Complejo Hospitalario de Soria, Gerencia Regional de Salud de \\ Castilla y León (Sacyl), Po Santa Bárbara s/n, 42005, Soria \\ 3 Universidad de Huelva. Dpto. de Psicología Social, Evolutiva y de la Educación; gumersindo.ti- \\ rado@dpsi.uhu.es \\ 4 National Distance Education University (UNED), Faculty of Psychology, Department of Social and Organi- \\ zational Psychology, C/ Juan del Rosal, 10, 28040, Madrid; cgarciaael@psi.uned.es; gtopa@psi.uned.es \\ * Correspondence: Correspondence: mllorente78@alumno.uned.es; Tel.: (+34-636-85-78-12)
}

Publisher's Note: MDPI stays neutral with regard to jurisdictional claims in published maps and institutional affiliations.

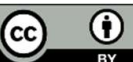

Copyright: (c) 2021 by the authors. Submitted for possible open access publication under the terms and conditions of the Creative Commons Attribution (CC BY) license (http://creativecommons.org/licenses/by/4.0/).

\begin{abstract}
The global pandemic produced by COVID-19 has caused serious consequences on people's physical health. In addition, the governments of the most affected countries have carried out containment measures to prevent the spread of the virus. These quarantine measures have caused the growing of mental health problems. The present work aims to carry out a descriptive analysis of the main psychiatric pathologies that have been admitted to a hospitalization unit in Soria (Spain), in 2019 and 2020. Besides, the differences between both years in duration of hospital stay, gender and age of the people admitted, or type of pathology have been tested. The results show that in 2019 there were 251 admissions with an average duration of 19.29 days (SD = 33.95). In 2020, 230 admissions with a duration of $12.07(\mathrm{SD}=16.49)$. The $t$ test for independent samples was significant $(t=$ $3.01 ; \mathrm{p}<.01)$ On the other hand, there were no differences in the number of women or men admitted. Regarding the type of pathology, significantly more schizophrenia, schizotypal and delusional spectrum disorders were detected in 2020 than in $2019\left(\chi^{2}=7.98, \mathrm{p}<.05\right)$. We conclude this research with a detailed analysis of the acute pathologies diagnosed in 2019 and 2020. The main limitations and future lines of research are discussed
\end{abstract}

Keywords: Psychiatric pathology; Mental Health; Covid-19; Hospital Admissions; Psychiatric Units

\section{Introduction}

The health emergency caused by the Coronavirus (Covid-19) has had serious consequences on the population's health and the economy at a global level. The quarantine measures have proved to be useful in lowering the dispersion of the virus, which has resulted in a lower incidence of the disease [1]. However, people in quarantine can experience a range of negative emotions and psychological consequences, such as boredom, loneliness and rage [2]. Along with the stress caused by the pandemic itself, these containment measures increase the levels of anxiety, depression and other symptoms related to stress [3].

A higher risk of infection with conditions such as cognitive impairment, poor risk awareness and small efforts regarding personal protection has been suggested in previously diagnosticated patients than in the overall population [4]. In addition, mental pa- 
tients may be more affected due to the emotional responses caused by the Covid-19 pandemic. This would cause relapses or worsening of their psychiatric pathology on account of their higher susceptibility to stress in comparison to the overall population [4]. Some authors have suggested that a rising of the psychosis cases can be predicted as a consequence of the Covid-19 health emergency [5].

A state of alarm with home confinement as its main measure was declared in Spain on March $13^{\text {th }} 2020$ and lasted a total of 98 days. The measures started to progressively reduce on May $4^{\text {th }}$. During a second wave of the virus, the government reenacted a state of alarm that limited the movement of people between regions and imposed a curfew in the entire country. In Castilla y León, the schedule of this curfew was between 22 p.m. and 6 a.m. In principle, this measure will remain until May $9^{\text {th }}$. All these prevention measures, alongside others like the closure of hotels and restaurants, gyms and shopping centers, have diminished social relations and forced certain routines of the people to change.

The present study intends to add scientific evidence of the impact of Covid-19 and the confinement measures on mental illnesses. The major purpose consists of describing the main characteristics of the hospital admissions that happened at the Psychiatric Hospitalization Unit of de Soria (Spain) in 2019 and 2020. It is aimed to analyze the differences between these years in order to analyze which mental health problems have worsened after the Covid-19 pandemic. It is also intended to discover if the duration of the hospital admissions have changed and if there were differences based on the gender or age of the patients.

\section{Materials and Methods}

The present research consists of a retrospective observational cohort study. The research team obtained the data of the patients admitted during 2019 and 2020 at the Psychiatric Hospitalization Unit of the Virgen del Mirón hospital in Soria (Spain), belonging to the Public Health Service of Castilla y León (Sacyl). The total of the sample was 481.

The following data was collected: psychopathology diagnosis according to ICD-10, duration of the hospital admission, gender and age. The years 2019 and 2020 were treated as absence and presence of the risk factor for mental health, meaning the Covid-19 pandemic. The data of both years from January to November (both included) was analyzed, since the data from December 2020 was not available for the research. The psychiatric diagnosis according to ICD-10 were classified by grouping the pathologies in the following categories:

F00: (F00-F09) Organic, including symptomatic, mental disorders.

F10: (F10-F19) Mental and behavioural disorders due to psychoactive substance use.

F20: (F20-29) Schizophrenia, schizotypal and delusional disorders.

F30: (F30-39) Mood [affective] disorders.

F40: (F40-49) Neurotic, stress-related and somatoform disorders.

F50: (F50-59) Behavioural syndromes associated with physiological disturbances and physical factors.

F60: (F60-69) Disorders of adult personality and behaviour.

F70: (F70-79) Mental retardation.

F80: (F80-89) Disorders of psychological development.

F90: (F90-F98) Behavioural and emotional disorders with onset usually occurring in childhood and adolescence.

F99: (F99) Unspecified mental disorder. 
The data was analyzed with the SPSS computer program. Descriptive analysis of the statistical data were conducted to know the frequencies and percentages of the variables. In addition, the qualitative variables were categorized to make contingency tables with Chi square analysis to test the hypothesis of non-association or independence between the variables. Regarding the psychiatric diagnoses, the most prevalent ones were selected and tested with contingency tables. In them, one variable was the year of admission to the hospital and the other was the diagnosis categorized in two groups (presence of absence of the most prevalent disorder). In addition, the Student $t$ test was carried out with the continuous dependent variables to compare a quantitative continuous variable between two different groups.

\section{Results}

In Table 1 we describe the descriptive statistics of the quantitative variables of the study.

Table 1. Descriptive statistics.

\begin{tabular}{|c|c|c|c|c|c|}
\hline Variable & $\mathbf{N}$ & Min. & Max. & $\mathbf{M}$ & SD \\
\hline \multicolumn{6}{|l|}{ Total sample } \\
\hline Duration of hospital stay & 481 & 1 & 270 & 15.84 & 27.26 \\
\hline Age & 464 & 11 & 95 & 49.34 & 19.62 \\
\hline \multicolumn{6}{|l|}{2019} \\
\hline Duration of hospital stay & 251 & 1 & 270 & 19.29 & 33.95 \\
\hline Age & 237 & 11 & 90 & 50.48 & 18.33 \\
\hline \multicolumn{6}{|l|}{2020} \\
\hline Duration of hospital stay & 230 & 1 & 206 & 12.07 & 16.49 \\
\hline Age & 227 & 12 & 95 & 48.15 & 20.86 \\
\hline
\end{tabular}

Regarding the qualitative variables, of the 481 participants, 246 were men and 235 women. In 2019, of the total of 251 admitted patients, 118 were men (47\%) and 133 women (53\%). In 2020, of the 230 patients admitted throughout the year, 128 were men (55.7\%) and 102 women (44.3\%). (See Figure 1).

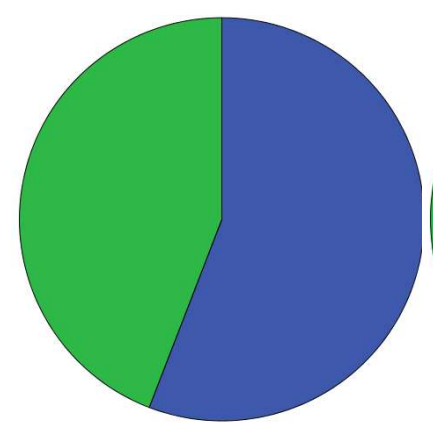

(a)

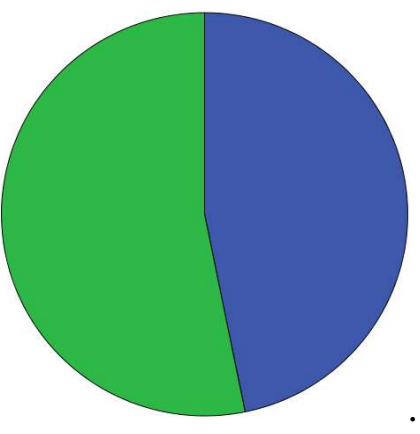

(b).

Figure 1. (a) Gender of participants (2020); (b) Gender of participants (2019).

Note: Green colour: Female; Blue colour: Male.

We also analyze the type of psychiatric diagnoses according to the ICD-10. In Table 2 , we present the frequencies in the type of diagnosis by year of admission. 
Table 2. Frequencies of psychiatric diagnoses by year according to ICD-10 (Classification of Mental and Behavioral Disorders).

\begin{tabular}{lcc}
\hline \multicolumn{1}{c}{ Psychiatric Diagnosis } & $\mathbf{2 0 1 9}$ & $\mathbf{2 0 2 0}$ \\
\hline \multicolumn{1}{c}{ (F00-F09) Organic, including symptomatic, mental disorders. } & 9 & 8 \\
(F10-F19) Mental and behavioural disorders due to psychoactive substance use. & 22 & 6 \\
(F20-29) Schizophrenia, schizotypal and delusional disorders. & 82 & 97 \\
(F30-39) Mood [affective] disorders. & 27 & 21 \\
(F40-49) Neurotic, stress-related and somatoform disorders & 16 & 11 \\
(F50-59) Behavioural syndromes associated with physiological disturbances and physical factors. & 3 \\
(F60-69) Disorders of adult personality and behaviour. & 4 \\
(F70-79) Mental retardation. & 58 \\
(F80-89) Disorders of psychological development. & 42 \\
(F90-F98) Behavioural and emotional disorders with onset usually occurring in childhood and adolescence. & 2 & 3 \\
(F99) Unspecified mental disorder & 0 & 0 \\
\hline
\end{tabular}

Note: Missing data: 2019 ( $\mathrm{n}=29) ; 2020(\mathrm{n}=13)$.

Next, we assess whether there are differences in the length of hospital stay between the two years. To do this, we made a comparison of means of the duration of the income between both years (2019 and 2020). Levene's test for equality of variances tells us that we can assume different variances $(F=10.52, \mathrm{p}<.01)$. The $t$ test for independent samples was significant $(t=3.01 ; \mathrm{p}<.01)$.

We analyze whether there are differences in the age of the patients admitted between the two years. We assume different variances according to Levene's test ( $\mathrm{F}=4.27, \mathrm{p}<.05)$. The $t$ test for independent samples was not significant $(t=1.27, p=.20)$.

Later, we evaluate whether there are differences in the number of women and men admitted between the two years. The contingency table and the subsequent Chi-square test were not significant $\left(\chi^{2}=3.58, \mathrm{p}=.058\right)$.

Finally, the most prevalent disorders in both years are (F20-29) schizophrenia, schizotypal and delusional disorders, (F30-39) mood disorders, and (F60-69) disorders of adult personality and behaviour. Significantly more schizophrenia, schizotypal and delusional spectrum disorders were detected in 2020 than in $2019\left(\chi^{2}=7.98, p<.05\right)$. There were no significant differences in mood disorders between 2019 and $2020\left(\chi^{2}=5.94, \mathrm{p}=\right.$ .051). There were significant differences in disorders of adult personality and behavior $\left(\chi^{2}\right.$ $=8.11, \mathrm{p}<.05)$. There were more diagnoses of personality disorders in 2019.

\section{Discussion}

The main characteristics of the hospital admissions that happened at the Psychiatric Hospitalization Unit of de Soria (Spain) during 2019 and 2020 has been described in the present study. A small decrease of the number of cases from 2019 to 2020 has been observed. This may be due to a lesser movement of people during the 98 days home confinement from March to May. Besides, a decrease of 7 days of the average duration of hospital admissions in 2020 compared to 2019 has been noticed. Besides that, there were no differences regarding gender or age of the admitted patients.

Regarding the type of pathologies of the patients admitted to the hospital, an increase of the psychotic breakdowns was detected in 2020. There were more cases of pathologies related to schizophrenia and schizotypical and delusional disorders than in 2019. These results support the evidence that the Covid-19 pandemic has generated an increase of the incidence of psychosis cases [5,6]. By contrast, other authors haven't found a rising of the psychotic experiences during the pandemic [7].

Regarding affective disorders, no differences have been noticed between 2019 and 2020. Other authors have reported results that didn't show a worsening of the state of mind of the mental patients during the Covid-19 pandemic [7]. In addition, self-reports 
from the patients suggested a higher wellness during the pandemic than before. Other authors have reported a reduction of the hospital admissions related to affective disorders in 2019 and 2020 (May to March) [8].

On another note, personality and behavioral disorders were prevalent in the 2019 admissions. However, studies that compare periods of time from both years (January to March) observed that the decrease of hospital admissions for personality disorders, schizophrenia spectrum and substance use disorders in 2020 was not statistically significant [8].

\section{1. Limitations}

The main limitation to this study was the size of the sample, because it may be unrepresentative of the overall population. Soria (40,000 inhabitants) is a small city and there might be significative differences with bigger population centers like Barcelona or Madrid. More retrospective studies that analyze the increase of the incidence of psychosis are encouraged.

It is also remarkable that during 2020 hospital admissions for acute psychiatric pathology patients with Covid-19 were treated at intern medicine units. This may have reduced the prevalence or duration of the admissions at the psychiatric unit. In addition, this study only analyzes data from the psychiatric unit admissions, excluding outpatient mental health care. Future lines of investigation could focus on carrying out studies that broaden the target population and include not only the severe psychiatric patients, but also the patients that require outpatient care.

Author Contributions: “M.LL-A., G.T., C.G-A. and G.T. contributed to the design and implementation of the research, to the analysis of the results and to the writing of the manuscript. All authors have read and agreed to the published version of the manuscript."

Funding: "This research received no external funding".

Conflicts of Interest: "The authors declare no conflict of interest."

\section{References}

Nussbaumer-Streit, B.; Mayr, V.; Dobrescu, AI.; Chapman, A.; Persad, E.; Klerings, I.; Wagner, G.; Siebert, U.; Ledinger, D.; Zachariah, C.; Gartlehner, G. Quarantine alone or in combination with other public health measures to control COVID19: a rapid review. Cochrane Database Syst. Rev. 2020, 9. https://doi.org//10.1002/14651858.CD013574.pub2

Park, S-C.; Park, YC. Mental Health Care Measures in Response to the 2019 Novel Coronavirus Outbreak in Korea. Korean Neuropsychiatric Association 2020. Psychiatry Invest 2020, 17, 85-6. https://doi.org//10.30773/pi.2020.0058

Giallonardo, V.; Sampogna, G.; Del Vecchio, V. et al. The Impact of Quarantine and Physical Distancing Following COVID-19 on Mental Health: Study Protocol of a Multicentric Italian Population Trial. Front Psychiatry 2020, 11, 533. https://doi.org//10.3389/fpsyt.2020.00533

Yao H, Chen JH, Xu YF. Patients with mental health disorders in the COVID-19 epidemic. Lancet Psychiatry, 2020, 7 (4): e21. https://doi.org//10.1016/S2215-0366(20)30090-0

Brown, E.; Gray, R.; Lo Monaco, S. et al. The potential impact of COVID-19 on psychosis: A rapid review of contemporary epidemic and pandemic research. Schizophr Res. 2020, 222, 79-87. https://doi.org//10.1016/j.schres.2020.05.005

Cowan, H.R. Is schizophrenia research relevant during the COVID-19 pandemic? Schizophrenia Research, 2020, 220, 271-272. https://doi.org/10.1016/j.schres.2020.04.002. 
Pinkham, A.; Ackerman, R.A.; Depp, C.; Harvey, P.D.; Moore, R.C. A Longitudinal Investigation of the Effects of the COVID-19 Pandemic on the Mental Health of Individuals with Pre-existing Severe Mental Illnesses. Psychiatry Research, 2020, 294. https://doi.org/10.1016/j.psychres.2020.113493.

Clerici, M.; Durbano, F.; Spinogatti, A.; Vita, G.; Girolamo, G.; Micciolo, R. Psychiatric hospitalization rates in Italy before and during COVID-19: did they change? An analysis of register data. Irish Journal of Psychological Medicine, 2020, 37, 283-290. https://doi.org/10.1017/ipm.2020.29 